\section{Literatur}

Bennett, M. J. D., Developing Intercultural Competence for Global Leadership, in: R.-D. Reineke, C. Fussinger (Hrsg.), Interkulturelles Management, Wiesbaden 2001, S. 205-226.

Bolten, J., Interkulturelle Kompetenz, Erfurt 2007.

Caligiuri, P., I. Tarique, R. Jacobs, Selection for international assignments, in: Human Resource Management Review, Vol. 19 (2009), S. 251-262.

Deardoff, D. K., Intercultural competence: Mapping the future research agenda, in: International Journal of Intercultural Relations, Vol. 48 (2015), S. 3-5.

Gröschke, D., Interkulturelle Kompetenz in Arbeitssituationen, München 2009.

Koester, J., M. W. Lustig, Intercultural communication competence: Theory, measurement, and application, in: International Journal of Intercultural Relations, Vol. 48 (2015), S. 20-21.

Leung, K., S. Ang, M. L. Tan, Intercultural Competence, in: The Annual Review of Organizational Psychology and Organizational Behavior, Vol. 1 (2014), S. 489-519.
Littrell, L., E. Salas, K., Hess, M. Paley, S. Riedel, Expatriate Preparation: A Critical Analysis of 25 Years of Cross-Cultural Training Research, in: Human Resource Development Review, Vol. 5 (2006), S. 355-388.

Morris, M. A., C. Robie, A meta-analysis of the effects of crosscultural training on expatriate performance and adjustment, in: International Journal of Training and Development, Vol. 5 (2001), S. 112-125.

Scheitza, A., Interkulturelle Kompetenz: Forschungsansätze, Trends und Implikationen für interkulturelle Trainings, in: $M$. Otten, A. Scheitza, A. Cnyrim (Hrsg.), Interkulturelle Kompetenz im Wandel, Band 1: Grundlagen, Konzepte und Diskurse, Berlin 2009, S. 91-119.

Thomas, A., Interkulturelle Kompetenz - Grundlagen, Probleme und Konzepte, in: A. Thomas (Hrsg.), Leben und Arbeiten in internationalen Kontexten. Schriftensammlung zur interkulturellen Kompetenz, Band 6, Berlin 2013, S. 140-177.

Thomas, A., Interkulturelle Handlungskompetenz. Versiert, angemessen und erfolgreich im internationalen Geschäft, Wiesbaden 2011.

\title{
Der Lebenslauf - Mut zur Lücke?
}

Unebenheiten im Lebenslauf sind nicht immer gleich ein Grund zur Sorge. Viele Lebensläufe weisen Lücken auf, die nicht automatisch zu schlechteren Chancen im Bewerbungsprozess führen. Wichtig ist der richtige und vor allem auch offene Umgang mit dem Thema:

\section{Arbeitslosigkeit}

Bei Personalverantwortlichen steht Ehrlichkeit hoch im Kurs. Nicht jede Lücke im Lebenslauf muss jedoch erklärt werden. Lücken von zwei bis drei Monaten sind nicht der Rede wert. Alles darüber hinaus kann den Personalverantwortlichen schon einmal zur Nachfrage ermutigen. Sind Lücken durch Arbeitslosigkeit bedingt gewesen, muss dies sogar im Lebenslauf angegeben werden. Bewerber können dabei aber durchaus auf Formulierungen wie ,berufliche Neuorientierung“، zurückgreifen. Ist man schon länger als ein Jahr auf Jobsuche, sollten unbedingt Sprachkurse oder anderweitige Weiterbildungen absolviert werden, um den Lebenslauf in dieser Phase aufzuwerten.

\section{Weltreise und Sprachurlaub}

Längere Reisen sollten Bewerber unbedingt im Lebenslauf erwähnen, denn diese gelten häufig als Erweiterung des
Erfahrungsschatzes. Zudem lassen sich während längerer Reisen die vorhandenen Fremdsprachenkompetenzen auffrischen. Auch eine Kulturreise wird von Personalverantwortlichen durchaus anerkannt.

\section{Auszeit durch Krankheit}

Längere Auszeiten durch Krankheit sollten ebenfalls im Lebenslauf erwähnt werden, falls man während der Zeit nicht erwerbstätig war. Bewerber sind allerdings nicht verpflichtet, das genaue Krankheitsbild zu beschreiben. Auszeiten durch schwere Schicksalsschläge oder psychische Erkrankungen müssen also nicht detailliert im Lebenslauf dargelegt werden. Auch bei konkreten Nachfragen eines Personalverantwortlichen darf getrost auf die Privatsphäre verwiesen werden.

\section{Literatur}

StepStone, Der Lebenslauf - Mut zur Lücke?, 2015, Online im Internet: URL: http://www.stepstone.de/Karriere-Bewerbungstipps/ der-lebenslauf-mut-zur-luecke.cfm (Abrufdatum: 17.09.2015).

Felix Horstmann, M.Sc., Marburg 